\title{
pXST, a novel vector for TA cloning and blunt-end cloning
}

\author{
Qin Liu', Hui-Jie Dang ${ }^{1}$, Yuan-Hang Wư ${ }^{1}$ Min Li ${ }^{1}$, Yin-Hua Chen ${ }^{1}$, Xiao-Lei Niu' ${ }^{1}$ Kai-Mian Li ${ }^{2}$ and Li-Juan Luo ${ }^{1 *}$
}

\begin{abstract}
Background: With the rapid development of sequencing technologies, increasing amount of genomic information has been accumulated. To clone genes for further functional studies in large scale, a cheap, fast and efficient cloning vector is desired.

Results: A bifunctional vector pXST has been constructed. The PXST vector harbors a Xcml-ccdB-Xcm/ cassette and restriction site Smal. Digestion the vector with $X \mathrm{cml}$ generates a single thymidine (T) overhang at $3^{\prime}$ end which facilitates TA cloning, and Smal gives blunt end that enables the blunt-end ligation. Multiple products with various sizes were amplified from cassava genome by PCR and each PCR fragment was separately cloned into a pXST using TA cloning and blunt-end ligation methods. In general, the TA cloning gave higher transformation efficiency than blunt-end ligation for inserts with all different sizes, and the transformation efficiency significantly decreased with increasing size of inserts. The highest transformation efficiency $\left(8.6 \times 10^{6}\right.$ transformants/ $\left.\mu \mathrm{g}\right)$ was achieved when cloning 517 bp DNA fragment using TA cloning. No significant difference observed in the positive cloning efficiency between two ligation methods and the positive cloning efficiency could reach as high as 100\% especially for small inserts (e.g. 517 and 957 base pairs).

Conclusions: We describe a simple and general method to construct a novel pXST vector. We confirm the feasibility of using PXST vector to clone PCR products amplified from cassava genome with both TA cloning and blunt-end ligation methods. The PXST plasmid has several advantages over many currently available vectors in that (1) it possesses $X \mathrm{cml}$-ccdB-Xcm/ cassette and restriction site Smal, enabling both TA cloning and blunt-end ligation. (2) it allows direct selection of positive recombinant plasmids in Escherichia coli through disruption of the $c c d B$ gene. (3) it improves positive cloning efficiency by introducing the $c c d B$ gene, reducing the possibility of self-ligation from insufficient digested plasmids. (4) it could be used by high performance and cost-effective cloning methods.

Therefore, this dual function vector would offer flexible alternatives for gene cloning experiments to researchers.
\end{abstract}

Keywords: PCR product, Cloning, T-vector, Blunt-end ligation, High efficiency

\section{Background}

Amplification and cloning of genes are fundamental techniques in the field of molecular biology. DNA amplicons by the polymerase chain reaction (PCR), require to be introduced into the plasmids for propagation, preservation, sequencing before transferred into expression vectors for the following functional characterization. The key step for cloning is to fuse the target DNA fragments into the linearized vectors, and many approaches have been developed

\footnotetext{
* Correspondence: luoljd@126.com

${ }^{1}$ Hainan Key Laboratory for Sustainable Utilization of Tropical Bioresources, Institute of Tropical Agriculture and Forestry, Hainan University, Haikou 570228, China

Full list of author information is available at the end of the article
}

to achieve this goal, including "TA" cloning, "blunt-end" cloning, TOPO, Gateway, etc. [1-5].

Generally, the currently available cloning methods can be divided into two categories, according to the critical enzymes catalyzed in the system: ligase-free methods and ligase-dependent methods. Most ligase-free methods achieved the DNA recombinant through DNA pairing and homologous recombination, which requires the whole corresponding systems, including the specific primers and enzymes, such as recombinase or topoisomerase I, thus, increasing the cost and complexity of the methods $[6,7]$. Due to the high cost and complicated protocol, hardly can commercially available ligase-free methods meet the requirement for large scale cloning and be used in most 
laboratories. Comparatively, the ligase-dependent methods are more widely and robustly used, which include cohesive-end ligation, blunt-end ligation and TA cloning [8]. The cohesive-end ligation links the target DNA fragments (inserts) to plasmids through complementary between the inserts and plasmid vectors, resulting in high recombinant efficiency. However, chances are high that the inserts contain multiple cloning sites, increasing the difficulties to choose the appropriate restriction enzymes. The blunt-end cloning walks around such problems by avoiding the digestion of the insert fragments. However new problems arise for the blunt-end cloning because the blunt ends of vectors could be self-ligated, decreasing the available vectors for ligating inserts. TA cloning seems to solve the problem of self-ligation by using the benefit of Taq polymerase. The Taq polymerase could preferentially add a single adenosine (dA) to the 3 '-end of a PCR product which is complimentary to the vectors with a single thymine overhang at 3 '-end (T-vector) [9]. Therefore, TA cloning is the simplest and most efficient approach for cloning of PCR products, because the method avoid the requirement of digesting the insert fragments and also the self-ligation problem of vectors.

After the transformation of the recombinant plasmids, an efficient selection method is required for the screening of positive colonies that contain the target inserts. Different strategies, including the white-blue screening, fluorescent protein-based method and introduction of antibiotic resistance and lethal genes, have been described [10-13]. The control of cell death B $(c c d B)$ gene encodes a potent inhibitor of gyrase that induces DNA breaking and cell death in the absence of the antidote gene $C c d A$ [14]. Such unique characteristic facilitates the positive selection of the recombinant plasmids, because only when the target
DNA fragments were inserted into vectors that disrupt the $c c d B$ genes, the cells could survive $[15,16]$.

In this study, we have constructed a novel cloning vector pXST by introducing the XcmI-ccdB-XcmI cassette and restriction enzyme site SmaI into the plasmid pMD-19 T. The pXST could be used by both TA cloning and blunt end ligation, providing more options when selection of cloning methods. In addition, introduction of $c c d B$ genes allows the direct selection of positive colonies and also reduces the self-ligation of blunt-end vectors. Furthermore, the system to catalyze the ligation is cheap and fast. Therefore, this plasmid is expected to be widely used as a high performance and cost effective cloning vector.

\section{Methods \\ Materials}

Plasmids pMD-19 T (simple) (TaKaRa, Dalian, China) and pDONOR221 (Invitrogen Corp., CA, USA) served as the backbone and $c c d B$ donor vector, respectively. Escherichia coli DB3.1 (Invitrogen) was employed to hold the destination vector. E.coli strain DH5a was used for the plasmid transformation and cloning. The LA Taq polymerase with GC buffer (TaKaRa) and Phusion DNA polymerase (Thermo Fisher, Waltham, MA) was employed to DNA fragment amplification. The PCR products purification and plasmid extraction kits were bought from Omega (Omega, Doraville, USA). T4 ligase and restriction enzyme $X c m I$ and SmaI were purchased from Thermo Fisher.

\section{Primer design}

All the primers used in this study were designed by the program Oligo7. The detail information of sequences and corresponding product lengths are listed in Table 1. Two $X c m I$ restriction sites were created by adding sequence $\left(5^{\prime}\right.$

Table 1 Primer sequences used in this study

\begin{tabular}{|c|c|c|}
\hline Name & Primer Sequences & Product size $(b p)$ \\
\hline$c c d B-F$ & CCAATACTTGTATGGgcagactggctgtgtataaggg & 487 \\
\hline$c c d B-R$ & CCAATACTTGTATGGctccggtctggtaagcacaac & \\
\hline ccdBseq-F & TCTITGCTGACGAGAACAG & $210+$ (insert) \\
\hline$c c d B s e q-R$ & CTITCATCCCCGATATGCAC & \\
\hline M13F & GTAAAACGACGGCCAGT & $126+$ (insert) \\
\hline M13R & CAGGAAACAGCTATGACC & \\
\hline F1 & GGCACAGACAAGATTGAACC & 517 \\
\hline R1 & AACACTGCAATTCCACGATG & \\
\hline $\mathrm{F} 2$ & TCCCGGTTGATTCTITACGTCT & 957 \\
\hline R2 & CGCAGTTTCCCATTTGTAATCGTC & \\
\hline F3 & TCCCGGTTGATTCTTTACGTCT & 1515 \\
\hline R3 & CAATCTTGTCTGTGCCCCGAA & \\
\hline F4 & CTITGCTTCCTATACCACGAGA & 2343 \\
\hline R4 & ACTAACACTGCAATTCCACGAT & \\
\hline
\end{tabular}

The underlined sequence is the specific restriction site for $X c m l$ and the small letters is the complementary sequence for the $c c d B$ amplification 
CCAATACTTGTATGG $3^{\prime}$ ) to the $5^{\prime}$ ends of primers to generate the $X c m \mathrm{I}-c c d B-X c m I$ cassette.

\section{Obtaining the $c c d B$ gene}

The pDONR221 vector was used as a template for the $c c d B$ amplification using $c c d B-F$ and $c c d B-R$ primers. The PCR reaction was performed in $50 \mu \mathrm{L}$ mixture consisted of $1 \mathrm{ng}$ pDONR221 vector, $0.4 \mu \mathrm{M}$ of each primer, $0.5 \mu \mathrm{L}$ TaKaRa LA Taq, $2.5 \mathrm{mM}$ dNTP mixture, $10 \times$ LA PCR Buffer I $\left(\mathrm{Mg}^{2+}\right.$ Plus) and an appropriate amount of $\mathrm{ddH}_{2} \mathrm{O}$. The thermic profile was set as follows: initial denaturing step at $95{ }^{\circ} \mathrm{C}, 30 \mathrm{~s}$ annealing at $56^{\circ} \mathrm{Cand} 30 \mathrm{~s}$ extension step at $72{ }^{\circ} \mathrm{C}$, followed by a final extension step at $72{ }^{\circ} \mathrm{C}$ for $5 \mathrm{~min}$. The PCR product was then purified by The E.Z.N.A. ${ }^{\circ}$ Gel Extraction Kit (Omega, cat. no. D2501) following the manufacturer's instructions.

\section{pXST construction}

The purified product was cloned into the pMD19-T (simple) vector to yield the pXST vector and the ligation was performed according to the manufacturer's instruction. The ligation mixture was used to transform competent $E$. coli DB3.1 cells. After the overnight culture, several clones were selected to detect the orientation of the inserted $c c d B$ fragment using the ccdB-F and M13R primers. The positive clone was cultured in liquid LB medium for overnight. Finally, the pXST plasmid was extracted using the Plasmid Mini Kit (Omega Bio-Tek, USA). The plasmid was digested with SmaI to generate the blunt-end fragments and XcmI to create T-vector.

The desired fragments were identified by agarose gel electrophoresis and purified by the Gel Extraction Kit (Omega). The pXST lethality was confirmed by transforming the vector into DB3.1 and DH5 $\alpha$ competent cells simultaneously and observing the bacteria growth.

\section{pXST transformation efficiency and positive cloning efficiency}

The DNA fragments of different lengths were amplified from the cassava MeSTP7 genomic sequence (Phytozome accession: Manes.03G180400). All fragments were amplified with or without 3 ' terminal A by using the $L A$ taq and Phusion DNA polymerase respectively. After the PCR products were purified, the ligation reaction was performed in $10 \mu \mathrm{L}$ volume using $50 \mathrm{ng}$ linearized pXST, $50 \mathrm{ng}$ purified fragment, $5 \mathrm{U}$ T4 DNA ligase, $1 \mu \mathrm{L} 10 \times \mathrm{T} 4$ buffer and

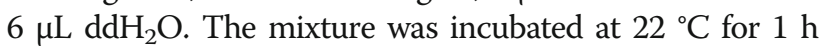
and then added into $100 \mu \mathrm{L}$ E. coli DH5 $\alpha$ chemically competent cells, followed by incubation on ice for $30 \mathrm{~min}$ and heat shock at $42{ }^{\circ} \mathrm{C}$ for $50 \mathrm{~s}$. All suspended cells were plated on a Luria-Bertani (LB)-agar plate with $100 \mu \mathrm{g} / \mathrm{ml}$ ampicillin. After an overnight culture the colonies were counted to calculate the transformation efficiency by using Calculator (http://www.sciencegateway.org/tools/transform.htm) and analyzed by colony PCR to examine the cloning efficiency. The mean values and standard deviation (SD) were calculated from the measurements of three independent experiments.

\section{Results}

The construction of the pXST cloning vector

The pXST vector was constructed followed the flow chart illustrated in Fig. 1. The XcmI-ccdB-XcmI cassette was amplified by using the pDONR221 vector as a template. The amplification was catalyzed by the polymerase TaKaRa LA Taq which could add an extra adenine to the $3^{\prime}$ end of the PCR fragments. The PCR products were examined by $1 \%$ agarose gel. As expected, and the electrophoresis showed a clear strap around the size of 500 bp. The target band was purified and then introduced into the commercial pMD19-T (simple) vector. The orientation of the insert fragment $c c d B$ gene was examined by performing colony PCR using the primer pairs, ccdB-F and M13R. After the colony PCR, the products were analyzed by gel electrophoresis (Fig. 2a). There were 6 out of 10 colonies showed a clear band, suggesting that the plasmids showing PCR amplification contain the $c c d B$ gene with the expected orientation (pXST) whereas the rest four without amplification have the $c c d B$ gene in the reverse orientation (pXST-R). The pXST and pXST-R were verified by sequencing. To examine if insertion of $c c d B$ gene in the reverse orientation would also lead to cell lethality, both pXST and pXST-R were transformed into E. coli DB3.1 ( $c c d B$ resistant strain) and $\mathrm{DH} 5 \alpha$, and the transformants were grew on LB plates with ampicillin selection independently. As showed in Fig. 3, DH5 $\alpha$ containing pXST can't grow on the LB-Amp plates with or without adding IPTG (Fig. 3b, c), suggesting that $c c d B$ gene can be well expressed driven by lac promoter even at basal level (without IPTG induction), and its expression caused the lethality. Whereas, DH5 $\alpha$ with pXST-R can grow on the LB-Amp plate with adding IPTG (Fig. 3d), which indicated that the insertion of $c c d B$ in the reverse orientation cannot be expressed, therefore, cannot result in lethality. These results also suggested that the $\operatorname{ccdB}$ gene fragment in pXST and pXST-R does not contain its own promoter, and its expression requires to be the driven of lac promoter. Together, the $c c d B$ gene in the pXST is expressed under the control of lac promoter and caused cell death in DH5 $\alpha$ but not in DB3.1.

\section{pXST transformation efficiency regarding PCR products}

Next, we continued to examine the feasibility of using pXST as T-vector and blunt-end vector for conducting both TA cloning and blunt-end ligation. The plasmid pXST was digested with SmaI to form a blunt-end vector and with $X c m I$ to generate the T-vector. The digested 


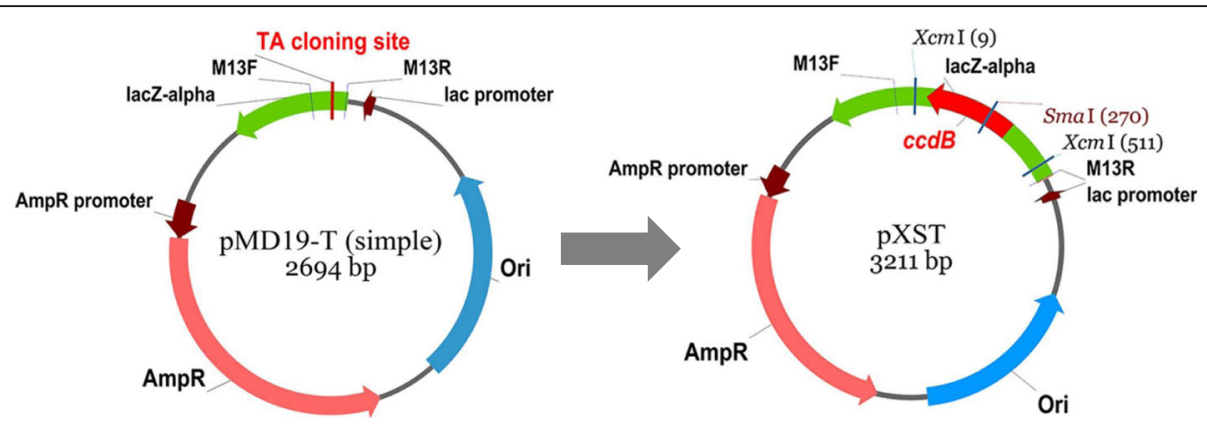

Fig. 1 Diagrammatic presentation of the pXST construction. $X c m l-c c d B-X c m l$ cassette is derived from the pDNOR221 vector. The recognition sites for $X \mathrm{cml}$ and $S \mathrm{mal}$ were indicated as italic. The lethal gene $c c d B$ was marked in red color

products were examined by electrophoresis, which showed the bands with the expected sizes (Fig. 2a). Target DNA fragments with different sizes (517, 957, 1515 and $2343 \mathrm{bp}$, respectively) were amplified from the cassava MeSTP7 genomic regions. In the PCR reaction, two different DNA polymerase was used, and one could add an additional adenine overhang at $3^{\prime}$ end of the PCR products, whereas the other could not. The two kinds of PCR products were cloned into T-vector and blunt end vectors generated from the $\mathrm{pXST}$ vector respectively. The transformation efficiency (TE) for different sized inserts were calculated for both TA cloning and blunt end ligation methods. The results showed that the TA

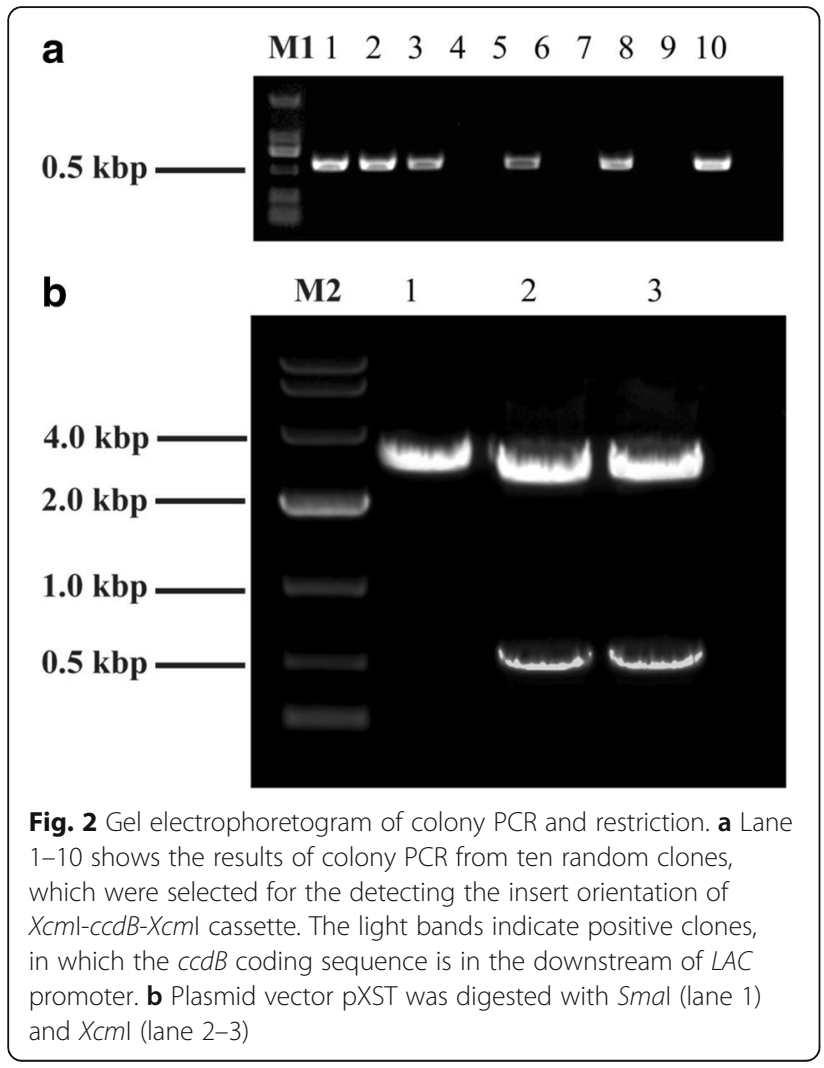

cloning generally gives higher TE compared to blunt end ligation for the insert fragments with all different sizes, and the efficiency decreased with the insert size increased (Fig. 4). The highest TE $\left(8.6 \times 10^{6}\right.$ transformants/ $\mu \mathrm{g}$ ) was obtained when the insert was $517 \mathrm{bp}$ in length. In addition, the positive cloning efficiency was calculated. For each condition, twenty random colonies were chosen to examine the positive insertion by PCR. The positive cloning efficiency was calculated by the ratio of recombinant versus the total number of selected colonies. Statistical analysis showed that the positive cloning efficiency for TA cloning and blunt-end cloning efficiency was both $100 \%$ for small inserts (517 bp and 957 bp) (Fig. 5). For the larger inserts, such as 1515 bp and 2343 bp PCR products, the positive cloning efficiency was decreased to $95 \%$. Mean values did not show statistically significant differences between the two ligation methods. These findings highlighted the fact that the pXST cloning system exhibited a strict selection of positive colonies.

\section{Discussion}

Though many commercial kits for DNA cloning are available, the high cost makes it difficult to meet the growing demands for gene cloning in large-scale. In the past years, various strategies have been reported to construct the homemade cloning vectors in laboratories. Most vectors are designed as T-vectors or blunt-end vectors because of theirs convenience in practice.

T-vector could be constructed by either adding a single thymidine residue to a blunt-end plasmid by transferase [17] or by restriction endonucleases, such as $\mathrm{Hpb}$ [18], $A b d \mathrm{I}$ [19], BfuI [20] and XcmI [21], which can recognize restriction sites containing variables nucleotide sequences, which provide a feasible way to construct T-vectors. Among above enzymes, XcmI has been widely used, because pUC-based plasmids which are the most popular vector series do not contain the restriction sites recognized by $X \mathrm{cmI}$. Therefore, addition of $X \mathrm{cmI}$ site to the pUC-based vectors would not disrupt their original 

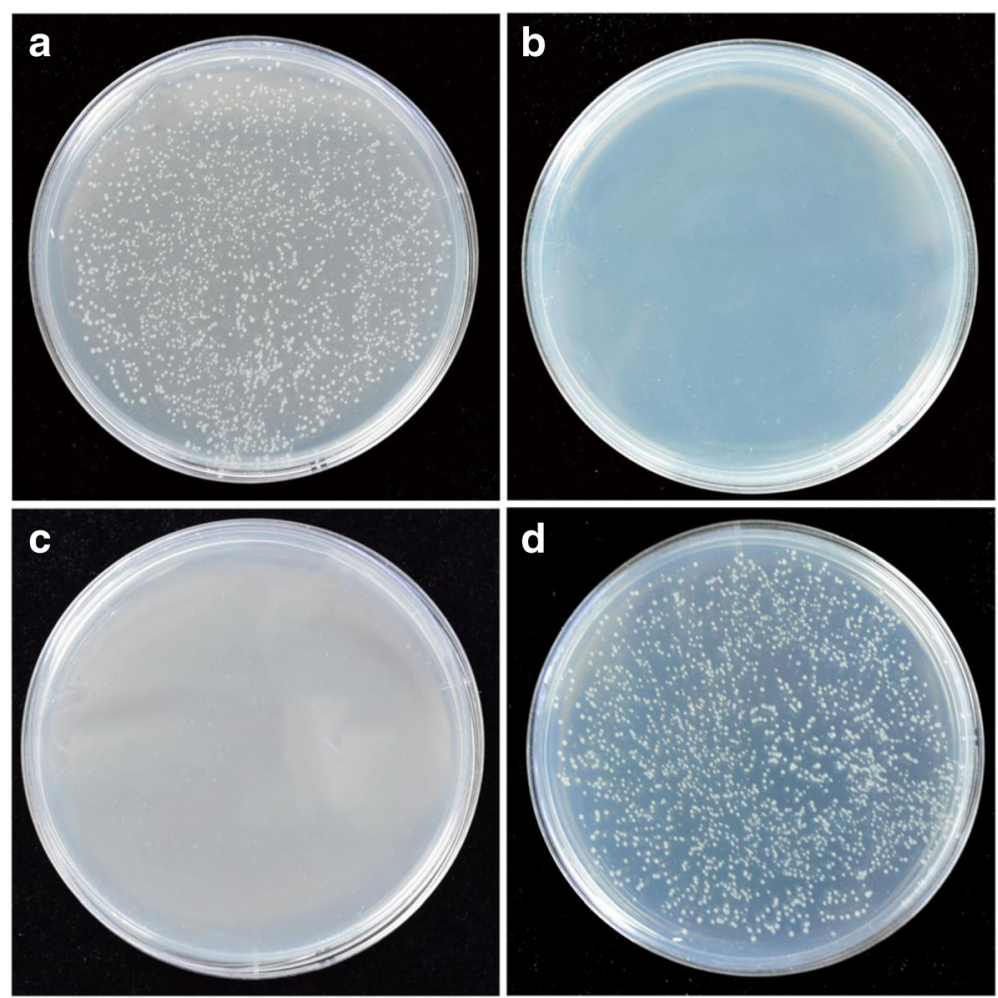

Fig. 3 The lethality testing of PXST and pXST-R. a Vector PXST was transformed into E.coli DB3.1 strain. DH5a strain harboring pXST was grown on the plates with (b) or without (c) IPTG respectively. $\mathbf{d}$ Vector pXST-R was transformed into DH5a and the strain survived in LB-plate with IPTG

features. However, T-vectors generated from restriction digestion would have self-ligation problems come from insufficiently digestion, especially when the size of the fragment between the two XcmI is too small [21]. Therefore, inserting a relative large DNA fragment within the two $X c m I$ sites seems to be an alternative choice to solve the problems mentioned above when designing the vector [22]. In the present study, we constructed the $X c m I-c c d B-X c m I$ cassette with length of 487-bp which is large enough for the $X c m I$ digestion. Furthermore, the $c c d B$ lethality can

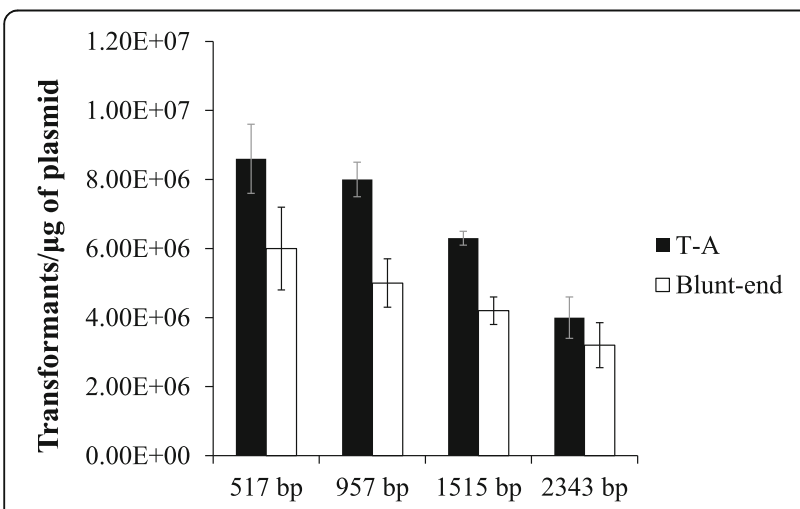

Fig. 4 Bacteria transformation efficiency for different sized inserts. Histograms represent average values and error bars mean standard deviations for three individual experiments guarantee that the transformants containing self-ligation vector would not survive, adding an additional layer of selection.

The blunt-end vectors have been frequently used for cloning DNA fragments since the DNA polymerases used to amplify DNA fragments have the high-fidelity due to the $3^{\prime}-5$ ' exonuclease activity and generate blunt-end fragments after the PCR reactions. Blunt-end vectors can

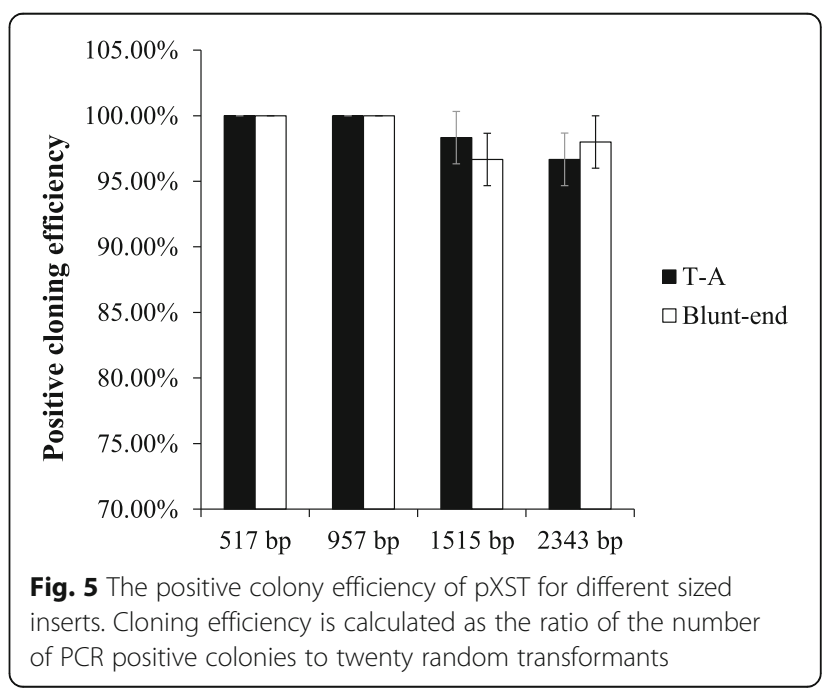


be generated by PCR or enzymes digestion. EcoRV and SmaI are the most popular endonucleases utilized to digest vector and produce blunt-end. Since the linearized vector and DNA fragment are both blunt-end in the T4 ligation reaction mixture, it causes the problem of vector self-ligation, reducing the possibility of successful recombinant and bringing great inconvenience in the following positive colonies screening. Although the dephosphorylation can be performed to minimize self-ligation of the linearized vector [23], it is still not an ideal solution as it is time consuming and costly. In this study, we introduced the SmaI site within the $c c d B$ gene. Therefore, if the vectors are self-ligated, the expression of $c c d B$ genes will result in cell death, reducing the possibility of the colony containing the self-ligated empty vectors, thus increasing the positive colony efficiency.

The pXST vector transformation efficiency regarding different sized PCR products was calculated by two ligation methods, TA cloning and blunt-end cloning. Generally, transformation efficiency is determined by a variety of factors, including the quality of competent cells, the molar ratio between the insert fragment and plasmid, and the ligation efficiency of the reaction mixture, etc. [24]. As high efficiency of transformation is not critical for cloning and subcloning purposes, a simple strategy that that can meet the minimum requirement for transformation without complex computation for the ratio of vector versus insert is more desirable. In this study, the transformation efficiency ranging from $3.0 \times 10^{6}$ to $8.3 \times 10^{6}$ transformants/ $\mu \mathrm{g}$ plasmid, was sufficient to meet the requirements of gene cloning and comparable to pELMO, which is a pUC18-based vector for TA cloning [25]. In addition, such efficiency could be reached even with equivalent amount of insert and pXST (50 ng for each, 1:1 ratio), increasing the convenience for practical experiments. The transformation efficiency of TA cloning is much higher than that of blunt-end ligation, which is due to the fact that T-A complement between the T-vector and the PCR fragment is easier for T4 ligation reaction to catalyze. For an ideal cloning vector, it should have a high positive cloning efficiency in addition to high transformation efficiency. The cloning efficiency of different size fragments between the two ligation methods was also tested in this study. The cloning efficiency was as high as $95 \%$ for both methods and there was no significant difference between the two methods. These results also supporting the idea that the introduction of $c c d B$ can reduce the possibility of self-ligation of vector.

Researchers usually require different plasmids to meet cloning requirements. In the present study, we constructed a bifunctianal vector pXST by combining XcmI, $S m a \mathrm{I}$ and $c c d B$ genes, which can be digested into linearized $\mathrm{T}$ carrier or blunt end vector. The vector pXST utilizes the $c c d B$ gene as a positive selection maker, saving the time in screening the positive colonies. For cloning vectors, they should have characteristics that allow the gene to be easily inserted into or removed. The pXST is designed without redundant cloning sites that would allow researchers to add any desired restriction enzyme sites to facilitate the subclone. In this study, the pXST was just designed for gene cloning, but the $X c m I-c c d B-X c m I$ cassette within the vector, which is proved to be a useful element in vector modification and deserved to be further used in the design of expression vectors. It would be serviceable in the plasmid constructions for various purposes, including gene over-expression, sub-cellular location, protein expression, etc.

\section{Conclusions}

In conclusion, a novel cloning vector pXST with high efficiency and low background is constructed in this study. The mechanism is easy to understand, and technique is simple to carry out. It is suitable for any lab to build this dual functional vector in with low cost.

\section{Abbreviations \\ Amp: Ampicillin; $c c d B$ : Control of cell death B; dA: Adenosine; IPTG: $\beta-D-1$ Thiogalactopyranoside; LB: Luria-Bertani; PCR: Polymerase chain reaction; TE: Transformation efficiency}

\section{Acknowledgements}

We would like to thank the anonymous reviewers for their helpful comments on the manuscript.

\section{Funding}

This work was supported by grants from National Natural Science Foundation of China (31660412), Core Research Budget of the Non-profit Governmental Research Institution (1630032016008), Funds of the modern agricultural industry technology system (CARS-HNCYH) and the Comprehensive Strength Promotion Plan of College in the Western Regions (M8K3124001001003002).

Availability of data and materials

The datasets supporting the conclusions of this article are available from the corresponding author on reasonable request.

\section{Authors' contributions}

$L-J L$ and $Q L$ designed the research. QL, H-JD, Y-HW, and ML. performed the experiments. QL, Y-HC, X-LN, K-ML, and L-JL. analyzed the data. All authors contributed to writing this manuscript and approved of its final manuscript.

Ethics approval and consent to participate

Not applicable.

Consent for publication

Not applicable.

\section{Competing interests}

The authors declare no conflict of interest.

\section{Author details}

${ }^{1}$ Hainan Key Laboratory for Sustainable Utilization of Tropical Bioresources, Institute of Tropical Agriculture and Forestry, Hainan University, Haikou 570228, China. ${ }^{2}$ Institute of Tropical Bioscience and Biotechnology, Chinese Academy of Tropical Agricultural Sciences, Haikou 570228, People's Republic of China. 
Received: 26 November 2017 Accepted: 9 July 2018

Published online: 13 July 2018

\section{References}

1. Clark JM. Novel non-templated nucleotide addition reactions catalyzed by procaryotic and eucaryotic DNA polymerases. Nucleic Acids Res. 1988;16(20):9677-86.

2. Aranishi F, Okimoto T. Engineered $X \mathrm{cml}$ cassette-containing vector for PCRbased phylogenetic analyses. J Genet. 2004;83(1):33-4.

3. Liu ZG, Schwartz LM. An efficient method for blunt-end ligation of PCR products. BioTechniques. 1992;12(1):28-30.

4. Wu WY, Gillies AR, Hsii JF, Contreras L, Oak S, Perl MB, Wood DW. Selfcleaving purification tags re-engineered for rapid Topo ${ }^{\circledR}$ cloning. Biotechnol Prog. 2010;26(5):1205-12.

5. Curtis MD, Grossniklaus U. A gateway cloning vector set for highthroughput functional analysis of genes in planta. Plant Physiol. 2003;133(2):462-9.

6. Bonsor D, Butz SF, Solomons J, Grant S, Fairlamb IJ, Fogg MJ, Grogan G. Ligation independent cloning (LIC) as a rapid route to families of recombinant biocatalysts from sequenced prokaryotic genomes. Org Biomol Chem. 2006;4(7):1252-60.

7. Zhang Y, Werling U, Edelmann W. SLiCE: a novel bacterial cell extract-based DNA cloning method. Nucleic Acids Res. 2012;40(8):e55.

8. Yao S, Hart DJ, An Y. Recent advances in universal TA cloning methods for use in function studies. Protein Eng Des Sel. 2016;29(11):551-6.

9. Eckert KA, Kunkel TA. DNA polymerase fidelity and the polymerase chain reaction. Genome Res. 1991;1(1):17-24.

10. Yang J, Xu J, Zhong X, Zhang Y, Wu Q, Li W, Zhang X, Zhen P, Yang $X$, Chen L. An advanced blue-white screening method for construction of shRNA expression vectors. Biochem Biophys Res Commun. 2009;390(1):97-102.

11. Kovach ME, Elzer PH, Hill DS, Robertson GT, Farris MA, Roop RM, Peterson KM. Four new derivatives of the broad-host-range cloning vector pBBR1MCS, carrying different antibiotic-resistance cassettes. Gene. 1995;166(1):175-6.

12. Park HK, Zeng C. Construction of an Xcml-generated $T$ vector bearing green fluorescent protein marker for direct cloning of PCR products. Anal Biochem. 2007;360(1):144-5.

13. Wang B, Zheng F, Quan J, Li X, Liu Y, Sun B, Zhang R, Guo G, Zhang J. A PCR-based technique to construct T-vectors for high-throughput cloning of PCR products. Anal Biochem. 2007;363(2):303-5.

14. Bernard $P$, Couturier M. Cell killing by the F plasmid CcdB protein involves poisoning of DNA-topoisomerase II complexes. J Mol Biol. 1992;226(3):735-45.

15. Wu C, Nerurkar VR, Yanagihara R, Lu Y. Effective modifications for improved homologous recombination and high-efficiency generation of recombinant adenovirus-based vectors. J Virol Methods. 2008;153(2):120-8.

16. Mandi N, Kotwal P, Padmanabhan S. Construction of a novel zero background prokaryotic expression vector: potential advantages. Biotechnol Lett. 2009;31(12):1905.

17. Holton T, Graham M. A simple and efficient method for direct cloning of PCR products using ddT-tailed vectors. Nucleic Acids Res. 1991;19(5):1156.

18. Mead DA, Pey NK, Herrnstadt C, Marcil RA, Smith LM. A universal method for the direct cloning of PCR amplified nucleic acid. Nat Biotechnol. 1991;9(7):657.

19. Jeung JU, Cho SK, Shim KS, OK SH, Lim DS, Shin JS. Construction of two pGEM-7Zf(+) phagemid T-tail vectors using Ahdl-restriction endonuclease sites for direct cloning of PCR products. Plasmid. 2002;48:160-3.

20. Janner CR, Brito ALP, Moraes LMP, Reis VC, Torres FA. pPCV, a versatile vector for cloning PCR products. Springerplus. 2013;2(1):441.

21. Jo C, Jo SA. A simple method to construct T-vectors using Xcml cassettes amplified by nonspecific PCR. Plasmid. 2001;45(1):37-40.

22. Gu J, Ye C. pYEMF, a pUC18-derived $X \mathrm{cml}$ T-vector for efficient cloning of PCR products. Mol Biotechnol. 2011;47(3):229-33.

23. Kovalic D, Kwak J-H, Weisblum B. General method for direct cloning of DNA fragments generated by the polymerase chain reaction. Nucleic Acids Res. 1991;19(16):4560.

24. Hanahan D. Studies on transformation of Escherichia coli with plasmids. J Mol Biol. 1983;166(4):557-80

25. Ramos AE, Munoz M, Moreno-Perez DA, Patarroyo MA. pELMO, an optimised in-house cloning vector. AMB Express. 2017;7(1):26.

\section{Ready to submit your research? Choose BMC and benefit from:}

- fast, convenient online submission

- thorough peer review by experienced researchers in your field

- rapid publication on acceptance

- support for research data, including large and complex data types

- gold Open Access which fosters wider collaboration and increased citations

- maximum visibility for your research: over $100 \mathrm{M}$ website views per year

At BMC, research is always in progress.

Learn more biomedcentral.com/submissions 